\title{
A Future Proof Optical Network Infrastructure for 5G Transport
}

\author{
Paola Iovanna, Fabio Cavaliere, Francesco Testa, Stefano Stracca, Giulio Bottari, \\ Filippo Ponzini, Alberto Bianchi and Roberto Sabella
}

\begin{abstract}
The telecommunication community has reached a broad consensus that current RAN and underlying transport will not be able to scale up to the traffic volume and quality expected in $5 \mathrm{G}$. Thus, it is mandatory to remove all the technological bottlenecks and operational rigidities to ensure a painless migration from the existing radio scenario to the $5 \mathrm{G}$ one.
\end{abstract}

This article presents a transport architecture able to serve as backhaul and fronthaul, to convey radio traffic on the same optical infrastructure. Cornerstones of the solution are: a novel photonic technology used to provide optical connectivity complemented with a dedicated agnostic framing; a deterministic switching module; a flexible control paradigm based on a layered scheme and on the slicing concept to facilitate optimal interaction of transport and radio resources while preserving a welldemarcated mutual independence.

Simulations and experiments are presented to demonstrate the aforementioned features.

Index Terms - Fiber Optics, Photonic Integrated Circuits, Radio Access Networks, Switches

\section{INTRODUCTION}

$\mathbf{T}$ The rising penetration of smart connected devices, promising compelling services anywhere and anytime, is having a huge impact on the mobile broadband infrastructure and on the real ability to provide quality of experience to the final users, whether humans or connected things.

5G new radio access ecosystem, expected by 2020 [1], will have to sustain an average traffic increased one order of magnitude and peak rates up to three orders of magnitude higher than current ones [2]. Many time sensitive services will also demand extremely low transmission latency.

Radio technologies will evolve by allocating new bands (beyond 10GHz), by leveraging higher order Multiple Input Multiple Output (MIMO), carrier's aggregation, and beamforming techniques. In parallel, the transport network, for Backhaul (BH) and Fronthaul (FH) applications, will demand support of higher capacities, to increase the number of transport clients, to support a wider range of performance requirements and to provide increased flexibility. This must be achieved in a cost-effective and sustainable manner.

Based on the radio architectures, it is possible to define many deployment scenarios, ranging from the fully centralized one, i.e. the Cloud Radio Access Network (CRAN), to the conventional scenario in which all functions are replicated at each radio site with monolithic Radio Base Stations (RBS). Moreover, as envisioned in [3], novel radio splitting models are under definition to meet $5 \mathrm{G}$ high bandwidth demands, leveraging different distribution of radio functions between radio unit nodes and centralized processing nodes. Realistic scenarios will see a mix of all said radio architectures, with a combination of traffic types to be transported among the radio devices.

In this paper, we define an Xhaul concept which can unify and enhance the traditional $\mathrm{BH}$ and $\mathrm{FH}$ segments by enabling a flexible deployment and the reconfiguration of network elements and networking functions. Xhaul sets connectivity services through the implementation of a control plane which provides a unified network model, supporting different underlying data planes and protocol split schemes.

Several implementations are possible for a Xhaul network. Our Xhaul solution is based on DWDM fiber rings connecting a central hub to remote nodes where radio and wireline clients are connected. Xhaul is enabled by a new type of photonic devices having a deep integration of optical functions on a single chip and presenting cost, footprint and power consumption adequate for this target network segment.

This paper is organized as follow: Section II defines the Xhaul network concept and illustrates the different radio transport needs. Section III describes the Xhaul architecture and its building blocks including optical transmission, framing, agnostic switching and control. Section IV provides an insight of the enabling photonic technologies. Section V reports analytics performance evaluations. Section VI illustrates a first practical demonstration of Xhaul in an experimental test bed built at Ericsson Research labs. Concluding remarks are given in section VII. 


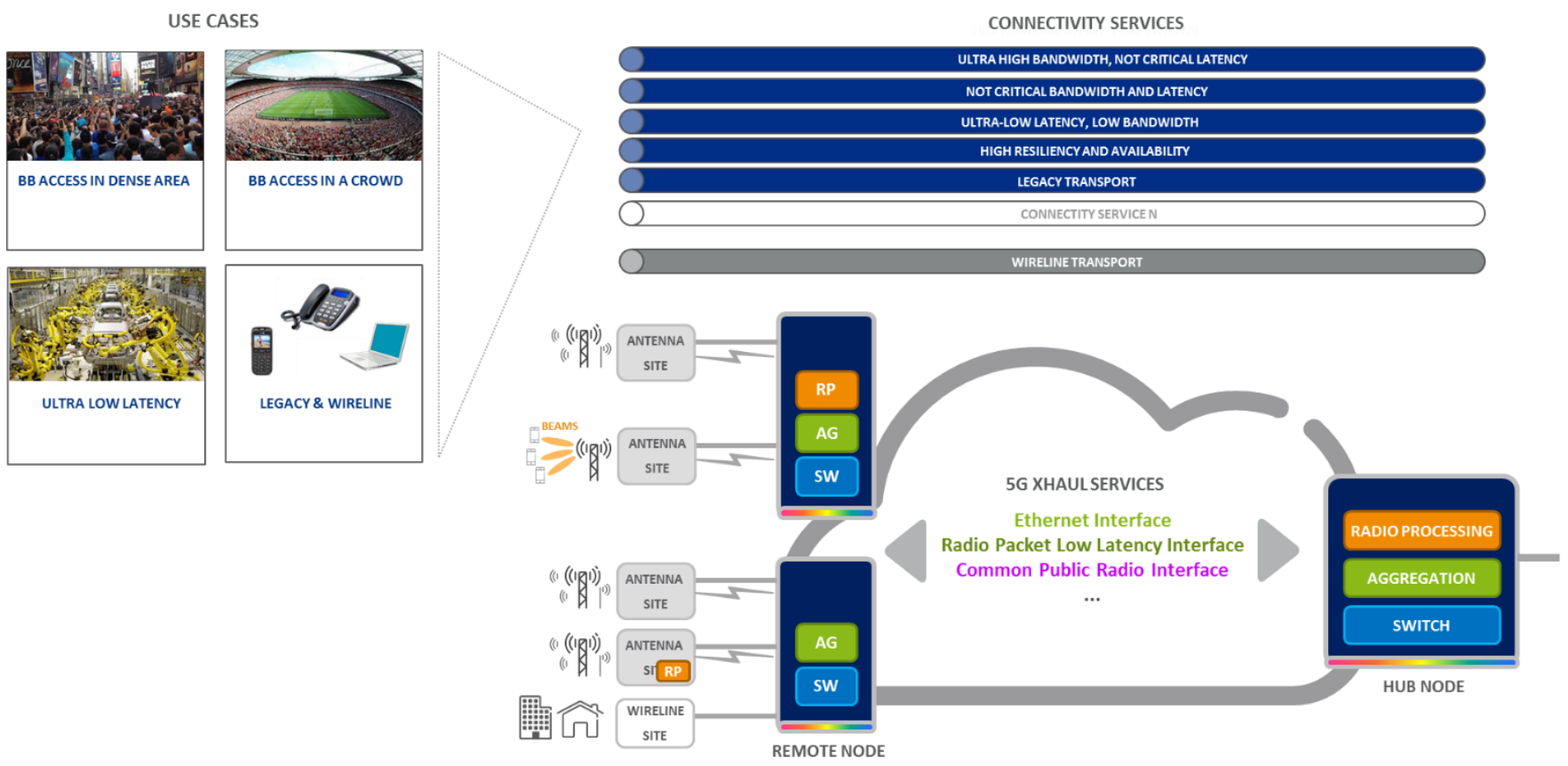

Fig. 1 - XHaul reference scheme

\section{The Xhaul Network CONCEPT}

In traditional Radio Access Networks (RAN), Radio Units (RU), performing radio functionalities, and Digital Units (DU), performing baseband processing, are integrated in a RBS which is typically backhauled by an Ethernet signal. Ethernet clients are transported across the BH segment. In CRAN architecture, Remote RU (RRU) and DUs are divided in two separated nodes and connected across FH network. RRUs are located at the antenna side. DUs are separated from RRUs and, possibly, aggregated in a DU pool. Centralizing DUs enables improved coordination of radio capabilities across a set of RRUs, faster RAN deployment, cost savings. Common Public Radio Interface (CPRI) [4] is the radio interface protocol widely used for communication between RRUs and DUs. Different mobile radio technologies can be transported by CPRI with different constraints on transport. For example, tolerated delay between RRU and DU (one way) is $\sim 200 \mu$ s for GSM/WCDMA traffic and $\sim 100$ $\mu$ s for LTE traffic. Other constraints are imposed on latency imbalance (asymmetry downlink/uplink) and jitter.

It has been extensively accepted that in $5 \mathrm{G}$ a new functional split [3] between DU and RRU will be required to mitigate the 5G bandwidth explosion. Some physical layer radio functions, e.g. resource mapping, will migrate to the RRUs. RRU will be in charge of generating the proper I/Q signals and radio carriers for the antennas. A new radio packet interface between DU and RRU will enable radio bandwidth of one order of magnitude higher, without a dramatic increase of the rate between DU and RRU compared with current CPRI. Moving towards a radio packet interface does not mean that conventional Ethernet switches can be used to connect DU and RRU, because the latency requirements will be still there and they will be carefully managed. This new Radio Packet Low-latency Interface (RPLI) is still under discussion and with different names from different vendors.

In Xhaul, both the $\mathrm{BH}$, CPRI FH and $5 \mathrm{G} \mathrm{FH}$, based on RPLI can benefit by a transport layer based on DWDM optical technologies which ensures low propagation delays, high throughputs, low power consumption, while being an economical choice in exploiting fiber resources. However, mixing all this interfaces cannot not be trivially considered as a problem of "tunneling" client traffics over dummy optical pipes. This is even truer if we consider a practical scenario in which mobile traffic can change over time and space, in distribution and load.

Another aspect that a cost effective network should consider, is that not all requirements are expected to be critical at the same time. For example, NGNM [1] has defined twenty-five use cases grouped in eight families.

The Xhaul infrastructure shall be open to provide different connectivity services in response to different radio needs. This concept is captured in Fig. 1.

On the left side of the picture some of the most prominent use cases expected in the $5 \mathrm{G}$ scenario are listed. On the right side of the picture it is illustrated a reference sketch of the Xhaul network. It is based on "Remote Nodes" where antennas are connected via wired or microwave links. In addition, wireline connections are connected to remote nodes. Xhaul is also based on a "Hub Nodes" which collects traffic from the several remote notes connected to the same Xhaul areas. The network in between is an optical network based on WDM technology.

In 5G systems, radio and transport networks will be abstracted into infrastructure slices. The slice is a connectivity service defined by a number of customizable 
software-defined functions that govern duration, capacity, speed, latency, robustness, security and availability, in a geographical coverage area. Through network slices, operators can provide, on demand, infrastructure as-aservice in a programmable way.

Slices are agnostic and independent of the underlying physical resources and on the used technologies. Infrastructure resources and functions can be dedicated to a slice or shared among multiple slices.

In this paper, a layered model is proposed where radio and transport have a client-server relationship. It allows both a tight interworking with radio and fixed access, while keeping the clear separation of responsibility, troubleshooting and services among radio and transport.

According to the layered model, it is possible to define classes of service at radio and transport layers identifying layer-specific requirements. Such a way, transport has some autonomy from radio to organize its internal resources in services and exposes them in corresponding slices. Transport can dynamically re-arrange traffic and provide sharing of physical resources for different slices view. It can also optimize the physical resources without complicating the radio tasks.

In Figure 1 some relevant 5G end user services are reported and, for each of them, it is shown the most critical requirement to consider. Broadband access in dense area and in a crowd, for example, will have the traffic density per area as critical parameter, possibly time bounded. Ultra-low latency applications, like the one in Machine Type Communications (MTC), will impose stringent limits to the end-to-end transmission delay without challenging bandwidth needs. Legacy mobile and wireline will also constitute use cases for Xhaul.

\section{ARCHITECTURE AND BUILDING BLOCKS}

Xhaul has a logical hub and spoke architecture which enables P2P logical connections between remote nodes and a hub node through fiber rings. This allows reducing the intermediate steps of processing of the signals thus limiting the latency required for transport. Any different physical topology where DWDM can be used to establish point-topoint connectivity through dedicated wavelengths, also applies to Xhaul. For example: linear chains of Optical Add Drop Multiplexers (OADM); point-to-multipoint distribution infrastructures, based on Wavelength-Selected or Wavelength-Distributed Passive Optical Networks (PON); meshed networks realized through Reconfigurable OADMs. Of course, implementation details, such as protection methods, vary accordingly.

Fig. 2 illustrates the topology. Many radio or wireline clients are connected to the remote nodes which act as entry points in the Xhaul network. This particular network arrangement allows better exploiting the concentration of the traffic and the statistical multiplexing, and allows a better traffic balancing among DUs. Daily and weekly variations, and "traffic tides" moving among a geographical area, open to significant optimization opportunities at the hub covering said area. For example, a single hub can serve a residential district and a business district which typical have complementary traffic trends during the 24 hours switching- on/off radio equipment with savings in power consumption and transport resources.

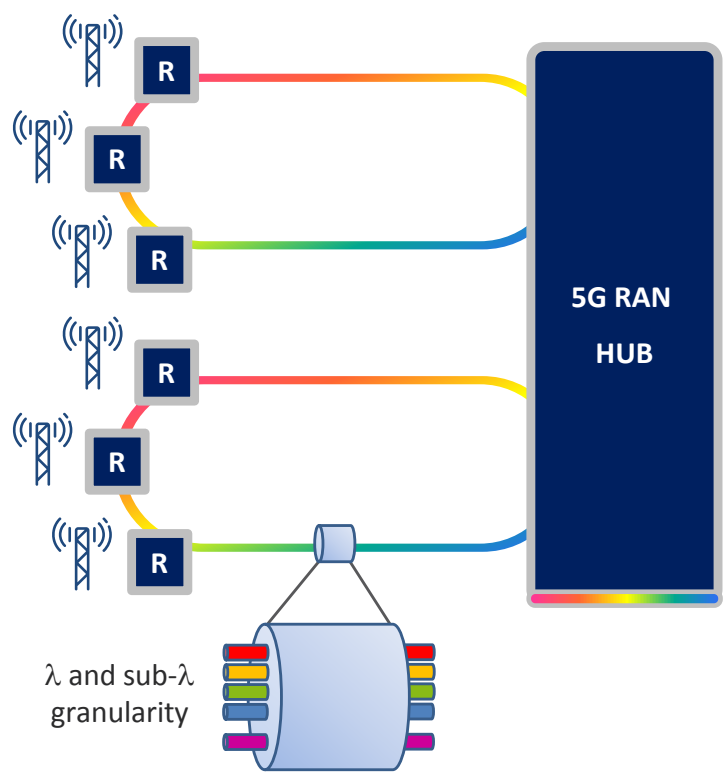

Fig. 2 - Xhaul topology

Important elements of Xhaul are the optical transmission and switching technologies with the agnostic framing, the deterministic switch and the overall Xhaul control. Following sections illustrates said elements in details.

\section{A. Optical Transmission}

The introduction of $5 \mathrm{G}$ mobile systems is pushing higher capacity $(100+\mathrm{Gbit} / \mathrm{s})$ in the fiber transport network.

As of distance requirement, coherent interfaces can achieve thousands of kilometers but the Xhaul segment spans to a maximum of few tens of kilometers so that direct detection interfaces would be more appropriate than coherent interfaces to lower cost and power consumption.

However, current $100 \mathrm{Gbit} / \mathrm{s}$ direct detection transceivers, e.g. based on PAM4, cannot achieve sufficient link budget in presence of a realistic number of optical add/drop nodes (OADMs) in the network. For example, a $20 \mathrm{~km}$ ring with one hub node and 8 OADM sites would require about $18 \mathrm{~dB}$ of link budget considering the following loss values: $0.6 \mathrm{~dB}$ for channels passing-through each OADM, $3 \mathrm{~dB}$ for added or dropped channel, $5.5 \mathrm{~dB}$ for wavelength multiplexer/demultiplexer at the hub and $0.25 \mathrm{~dB} / \mathrm{km}$ fiber attenuation coefficient.

Fiber chromatic dispersion is another issue with $100 \mathrm{Gbit} / \mathrm{s}$ DWDM direct detection: in a Xhaul network it is desirable to avoid dispersion compensation in line, to save cost and not to introduce additional loss, but direct detection interfaces cannot exploit electrical equalization like coherent interfaced.

A practical solution to increase link budget and chromatic dispersion tolerance is to transmit $50 \mathrm{Gbit} / \mathrm{s}$ wavelengths instead of $100 \mathrm{Gbit} / \mathrm{s}$ ones. While this halves the aggregate capacity, the total capacity value (a few Terabit/s) is still sufficient for $5 \mathrm{G}$ transport. 
In summary, an ideal modulation format for Xhaul should satisfy the following features:

- Direct detection, to decrease cost and power consumption, avoiding local oscillator and digital signal processing at the receiver;

- No dispersion compensation up to $20 \mathrm{~km}$;

- Receiver sensitivity sufficient to achieve a link budget of about $18 \mathrm{~dB}$, with or without optical amplification.

The following Fig. 3 compares direct detection modulation formats at $50 \mathrm{Gbit} / \mathrm{s}$ as regards the chromatic dispersion tolerance. It is obtained by numerical simulation considering $0 \mathrm{dBm}$ of channel power transmitted in a Standard Singlemode Fiber (SMF) with $0.22 \mathrm{~dB} / \mathrm{km}$ and $-20 \mathrm{ps}^{2} / \mathrm{km}^{-1}$ fiber attenuation and chromatic dispersion coefficients, respectively. The fiber is modeled as a linear system, since non-linear effects are negligible, due to low transmitted power and short distances in the Xhaul network segment. The optical penalty on the $\mathrm{y}$-axis is defined as receiver sensitivity referred to the On-Off Keying (OOK) sensitivity at $0 \mathrm{~km}$ and $10^{-3} \mathrm{BER}$, a value that can be corrected by common hard-decision Forward Error Correction (FEC) codes.

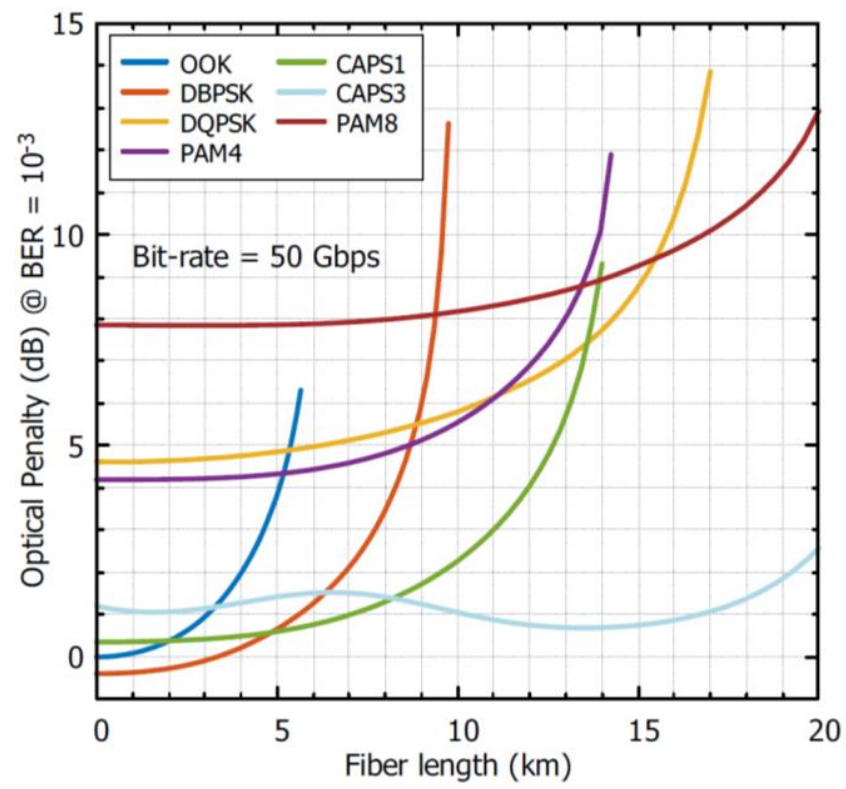

Fig. 3 - Direct detection modulation formats at $50 \mathrm{Gbit} / \mathrm{s}$

Differential Binary Phase Shift Keying (DBPSK) and Differential Quadrature Phase Shift Keying (DQPSK) are directly detected by an interferometric receiver with balanced photodiodes.

Combined Amplitude Phase Shift (CAPS) modulation formats family is described in [5]. CAPS- 1 is indicated simply as CAPS. At the transmitter, CAPS-N requires a $2^{\mathrm{N}}$ states digital encoder followed by a IQ modulator, with the exception of CAPS-1 which use a simple Mach Zehnder Modulator (MZM). At the receiver, all CAPS formats are directly detected as OOK, with no need of digital signal processing.

Fig. 3 shows that CAPS-3 outperforms the other modulation formats for link distances higher than $8 \mathrm{~km}$.

Discrete Multi-Tone (DMT) is an additional modulation format, using direct detection. Its performance is very implementation specific, depending of design variable (number of sub-carriers, cyclic prefix, pilot tones...). This is why DMT has not been included in Fig. 3.

Fig. 4 reports experimental results (dots) versus simulations (curves), comparing OOK, PAM-4 and CAPS-3 at $25 \mathrm{Gbit} / \mathrm{s}$ (it was not possible to perform $50 \mathrm{Gbit} / \mathrm{s}$ experiments with the available instrumentation). The experiments confirm CAPS-3 robustness to chromatic dispersion. To obtain results in Fig. 4 a Mach Zehnder (MZ) modulator was used to generate OOK and PAM-4 signals and a nested-MZ IQ modulator was used to generate the CAPS-3 signal. The electrical signals at modulator's input are obtained by means of a DAC with $13 \mathrm{GHz} 3 \mathrm{~dB}$-bandwidth, operating a 64 GSample/s. The digital signal, at the input of the DAC, is generated through off-line digital signal processing by encoding a periodic pseudo-random binary sequence (BRBS) of length $2^{11}-1$, according to the considered modulation format.

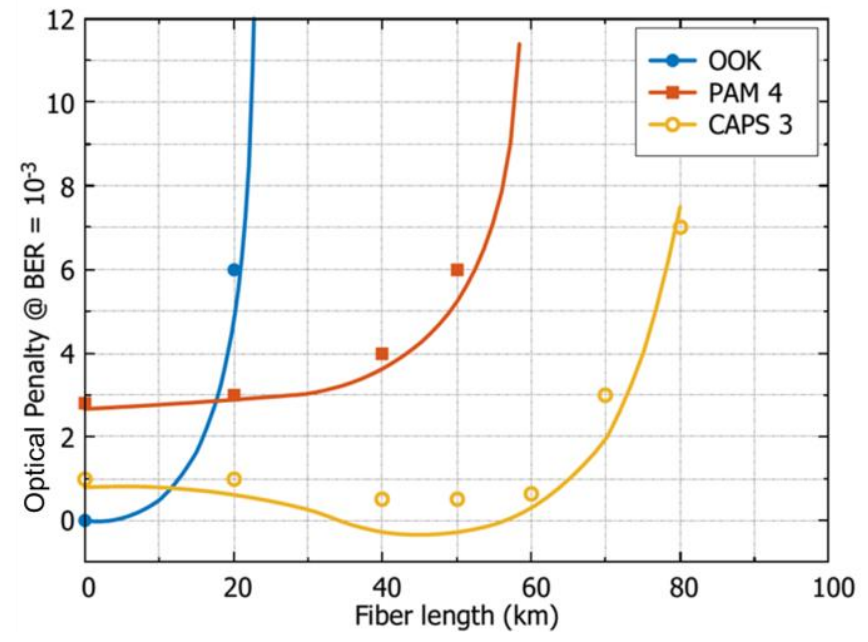

Fig. 4 - Modulation formats comparison at $25 \mathrm{Gbit} / \mathrm{s}$ : experiments (dots) vs. simulations

\section{B. Framing}

Ethernet, CPRI or RPLI clients can be separately mapped over dedicated optical channels in the DWDM comb. A set of optical channels is used to transport Ethernet traffic originated by RBSs. A second set is used to transport CPRI traffic between RRUs and DUs. Finally, a third set is used to transport a mix of Ethernet and CPRI traffic, wrapped in a novel framing structure. For illustration purposes, the following discussion will refer to CPRI but these considerations can be extended to RPLI or any kind of time sensitive client signals.

The use of dedicated optical channels ensures easier management and sharp client type segregation, facilitating shared/leased network scenarios. The use of shared $\lambda \mathrm{s}$ enables better bandwidth utilization and a fewer number of optical transceivers.

Sharing is enabled by a simple and novel framing, providing FEC and Operation and Maintenance (OAM) for both CPRI and Ethernet, without the complexity and the synchronization performance degradation introduced by standard protocols like OTN. 
(a)

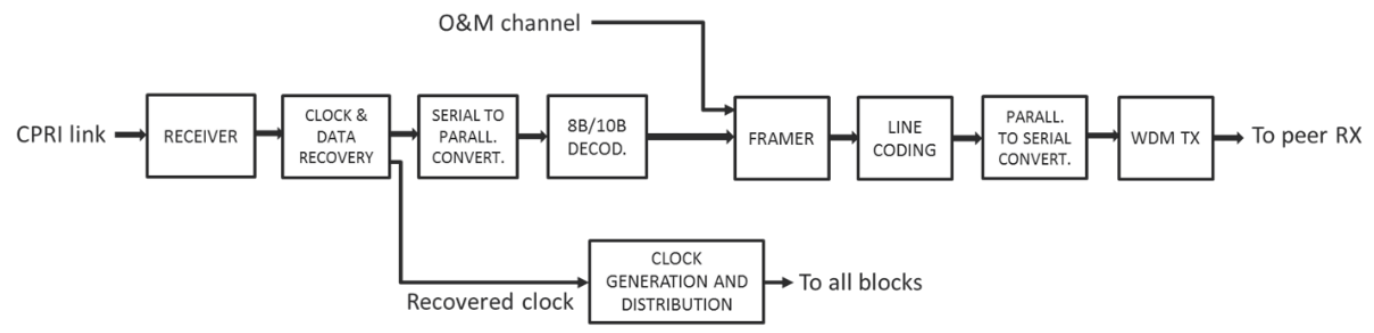

(b)

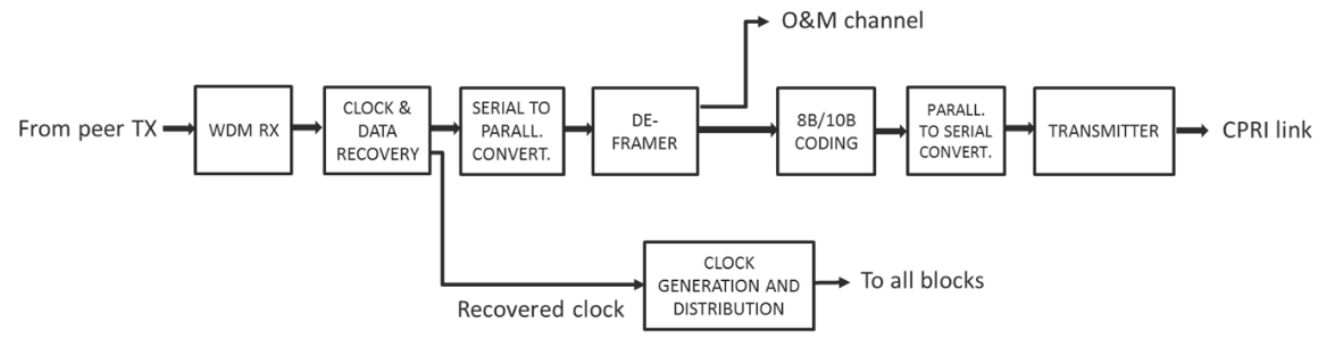

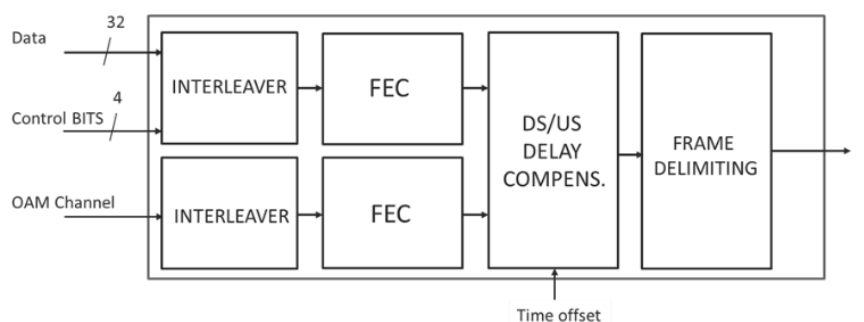

(c)

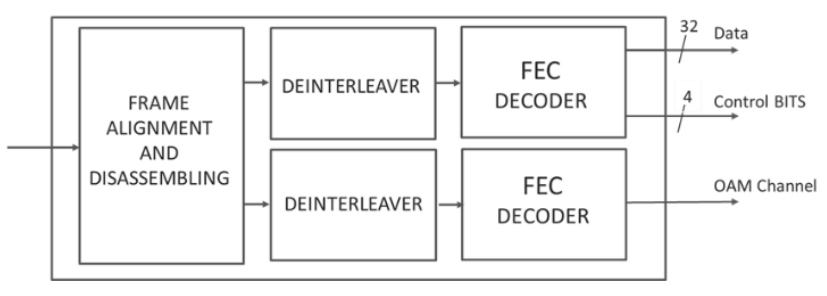

(d)

Fig. 5 - (a) Transmitter, (b) Receiver, (c) Framer, (d) Deframer

OTN [6] is an optical transport standard developed by the ITU-T. It is also known as ITU G.709 and "digital wrapper". In OTN, the ITU defined payload encapsulation, OAM overhead, forward error correction (FEC) and multiplexing hierarchy. The result is a transport standard that includes the benefits of SDH (such as resiliency and manageability) but with the improvements for transporting data payloads. OTN standards includes a standard multiplexing hierarchy, defining exactly how the lower rate signals map into the higher-rate payloads. This allows any OTN switch and any WDM platform to electronically groom and switch lower-rate services within $10 \mathrm{Gbit} / \mathrm{s}, 40 \mathrm{Gbit} / \mathrm{s}$, or $100 \mathrm{Gbit} / \mathrm{s}$ wavelengths.

CPRI mapping over OTN has been recently included in the ITU-T supplement [7]. The main challenge of transporting CPRI, and any time sensitive FH interface, over OTN is to limit the jitter and wander introduced while mapping and demapping CPRI to OTN. An analysis on Root Mean Square of the frequency offset ("jitter") and Mean Time Interval Error has been performed by ITU-T. ITU-T simulations show that to meet $2 \mathrm{ppb}$, as specified by the CPRI standard, stringent de-synchronizer bandwidth is required, much lower than 300 $\mathrm{Hz}$ normally used in OTN. This would lead either to design RRUs capable to tolerate higher input noise or to redesign the OTN equipment including very stable oscillators and sharp filters. Another issue, still under discussion, is the compensation of a possible imbalance of latency times in up- and down- stream. Today the practical use of CPRI over OTN is limited to the case of synchronous mapping of CPRI signals belonging to a single synchronization domain.

To overcome the issues that arise when mapping time sensitive FH interfaces over OTN, an alternative multiplexing methodology is presented in the following. Furthermore, it is described how the methodology can be extended to Ethernet client signals. Though the discussion focus is on optical channels, the methodology is agnostic to the propagation medium and can be applied to wireless signals as well. For a cost effective implementation, the proposed method makes the realistic assumption that $\mathrm{FH}$ signals are transported over short reach links (a few tens of kilometers) so that there is no need for advanced features such as the complex multiplexing hierarchy and protection mechanisms.

The proposed framing procedure is synchronous to the CPRI client to avoid any degradation of the synchronization accuracy.

Optional FEC is provided, based on RS $(255,239)$ but the number of interleaved codecs can optionally be reduced to limit the additional latency. First experiments have shown a FEC latency lower than $4 \mu$ s with 9 interleaved codes, with no appreciable degradation of the FEC gain with respect to the OTN case.

The bandwidth efficiency compared to CPRI is improved replacing spectrally inefficient line codes, as $8 \mathrm{~B} / 10 \mathrm{~B}$, code 
with more efficient scramblers, e.g. using as generating polynomial $1+\mathrm{x}+\mathrm{x}^{3}+\mathrm{x}^{12}+\mathrm{x}^{16}$, leaving space to FEC overhead and in-band signaling.

The transmitter scheme is outlined in Fig. 5 (a), taking CPRI Option-7 (9.8304 Gbit/s) as an example.

The clock signal is extracted from the received CPRI signal and distributed to all the transmitter blocks. After serial to parallel conversion, the $8 \mathrm{~B} / 10 \mathrm{~B}$ redundant bits are removed with the exception of the control bits which identify the Kcodes. Then, a framer block Fig. 5 (c) applies FEC to data, control and any other OAM bit. If desirable, different FEC codes or interleaving could be used for data and OAM bits. The framer includes also a block for the compensation of the difference between upstream and downstream delays, as required by the CPRI client. This can be done by means of a buffer. Finally, the assembled frame is scrambled.

The inverse operation is performed at the receiver Fig. 5 (b) with de-framer Fig. 5 (d).

An example of frame structure is illustrated in Fig. 6.

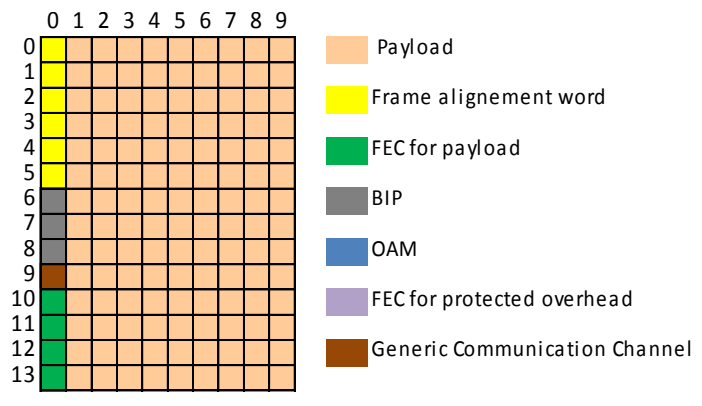

rows 14 to 149

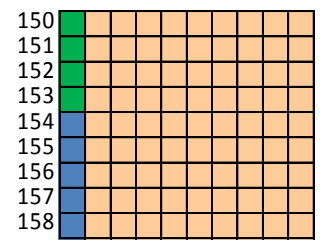

rows 159 to 218

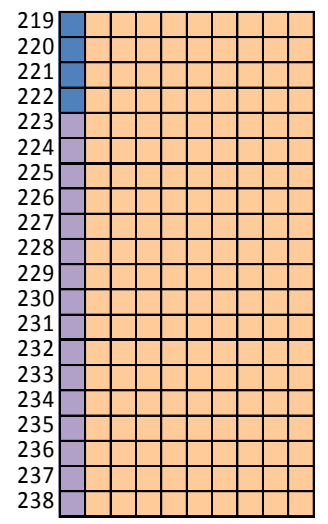

Fig. 6 - Example of frame structure (239 rows by 10 columns).

The frame is 2390 octets long, arranged in 239 rows by 10 columns. Columns 1 to 9 are for payload while column 0 is reserved for overhead: Frame alignment word (distributed in rows 0 to 5), FEC codes for payload (rows 10 to 153), Bit Interleaved Parity (rows 6 to 8), Generic communication channel (row 9), OAM channel (rows 154 to 222), FEC code for protected overhead (rows 223 to 238).

When number and bit rate of the $\mathrm{FH}$ client signals are not sufficient to "fill" a wavelength up to the maximum supported bit rate, the unutilized bits can be used to transport other type of clients. Especially Ethernet is of interest so that the same DWDM channel can serve $\mathrm{FH}$ and $\mathrm{BH}$ connections.

The proposed frame cannot provide all the set of features ensured by OTN but it is intended to target a simpler scenario, where point-to-point logical connections are the most frequent case.

The basic concept is very simple and consists of allocating two separate portions of the same frame to Ethernet and CPRI (or CPRI-like) clients. Size and position of the portions within the frame are known as well as the frame size, making very easy to separate Ethernet packets from CPRI frames.

Portion size and position can be programmable, e.g. via control interfaces, depending on network configuration and planned traffic load. The frame is synchronous to the CPRI clock in order to minimize the impact on delay and jitter sensitive CPRI frames while ingress Ethernet packets are buffered to absorb differences in clock value and accuracy. A possible way to map Ethernet frames in the dedicated timeslots of the frame is the use of the Generic Framing Procedure (GFP).

\section{Switching}

The reference scheme of the Xhaul switch is illustrated in Fig. 7. Ethernet and RPLI clients, demanding low and high latencies respectively, are first sent to a packet scheduler complemented with a set of buffers for time alignment. This block can be based on conventional Ethernet switch technology, assigning different priorities to different latency classes. Several implementations are possible: describing them would be out of scope of the paper. Another block is in charge of multiplexing CPRI clients, according to the multiplexing protocol described in the CPRI specification [4]. The time slotted switch maps all Ethernet, CPRI, and RPLI clients according to the agnostic framing described in Section III.B. Framed traffic is then wrapped in optical channels and sent towards the hub via the optical transport network. This can be performed by ROADMs, based on standard technologies or new technologies (described in Section IV). 


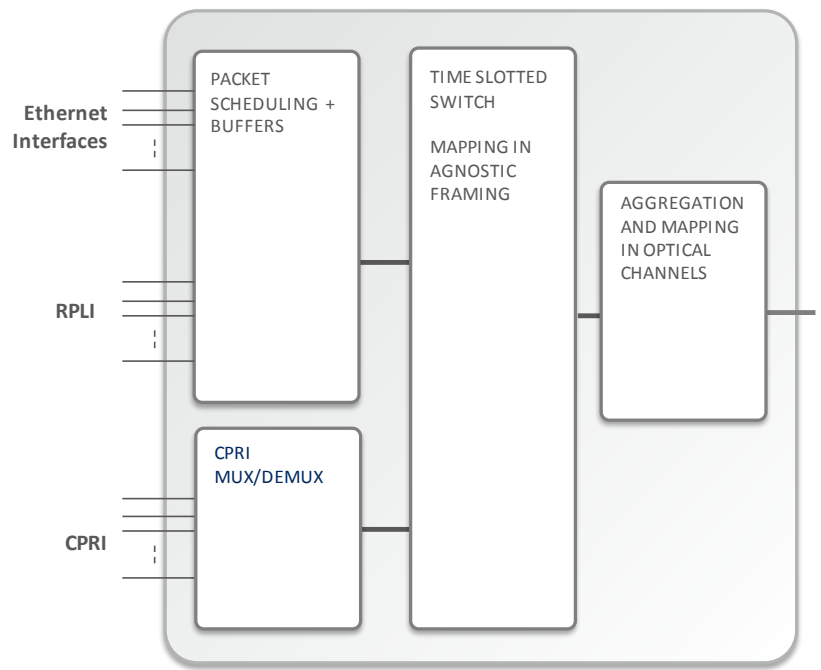

Fig. 7 - Switch reference scheme

The proposed switch architecture allows to solve the issues that would arise with conventional packet or circuit switches. Although, in principle, packet-based interfaces are appealing for Xhaul applications because they promise to manage both Ethernet and CPRI in the same off-the-shelf packet switching engine, Packet Delay Variation (PDV) and delay control are difficult to manage for long chain of switches or as soon as the ingress traffic approaches the switch overload condition. IEEE 802.1 Time-Sensitive Networking (TSN) [8] is studying this problem but there are issues with latency (in case of multiple switching hops), with deterministic delay to be ensured downstream and upstream, with PDV, and with synchronization distribution.

On the other hand, circuit switches can better deal with jitter and delay control but loses any statistical multiplexing gain, which is a fundamental feature to deal with $5 \mathrm{G}$ traffic loads. Statistical multiplexing is indeed highly beneficial in 5G that is characterized by a bursty distribution of traffic where peaks can be as high as $10 \mathrm{Gbit} / \mathrm{s}$ per sector and average as low as $200 \mathrm{Kbit} / \mathrm{s}$. Without statistical multiplexing, a network serving 100 sectors would require 1 Tbit/s.

\section{Control}

Xhaul is a new transport network segment with specific requirements, compared with the traditional access and metro where the level of dynamicity is limited and overprovisioning is typically applied. As explained in Section II, the control of the Xhaul network benefits from a layered model (slicing), through which the Xhaul control handles the transport resources by dynamically mapping the traffic, organizing the slicing view for the RAN. The layered model also guarantees clear demarcation points between the Xhaul network and its client RAN.

Fig. 8 illustrates a schematic view of the control functions, showing a radio controller managing radio equipment (i.e. RRUs, DUs, RBSs) and a Xhaul controller for the transport network. In this model, the radio layer is extended with a new Radio Logic (RL) block which receives requests to set $5 \mathrm{G}$ services, translates them in requests for radio and to Xhaul controllers, as described in the layered slicing model and performs radio-transport orchestrating function.

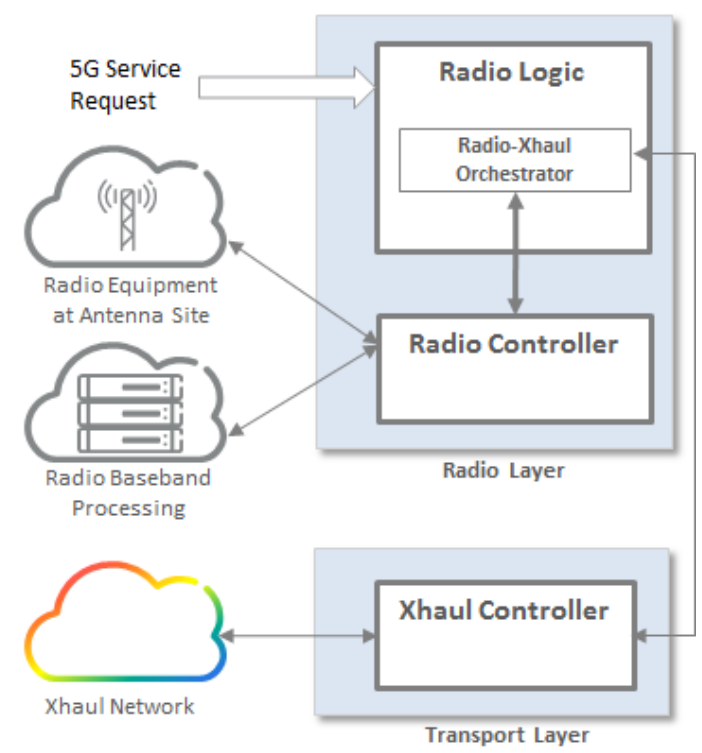

Fig. 8 - Control architecture

For example, the RL may receive a traffic matrix with end user mobile connection requests and service parameters. On this basis, the RL determines which RRU or RBS shall be connected to the hub defining the connectivity and its parameters (e.g. bandwidth, service type, resiliency level, policy constraints).

The RL determines if coordination requirements impose to use the same path for different connected RRUs. For example, if some cells require very tight phase alignment, as in MIMO, then the relevant FH flows shall be transported together minimizing the phase misalignments. RL also determines which high bandwidth traffic demand needs to be distributed on multiple optical channels and which are the aggregation policy constraints.

The Xhaul controller receives the transport demands, grouped in traffic matrixes, and determines the best possible path to serve each demand at the given time or in a planned time interval. For example, the Xhaul controller defines: the mapping of data traffic as described in Section IIIB; the aggregation in optical wavelengths; and the configuration of the switch described in Section III.C.

The Xhaul controller can split and recombine correlated radio requests over several optical channels (multiwavelength transport). For example, even if it is preferred that coordinated radio traffic is conveyed on the same wavelength, different wavelengths can be considered if the radio constrains, e.g. latency, are satisfied.

As another example, support of time-sensitive traffic can require the Xhaul controller to re-route non-time-sensitive traffic on alternative paths.

Finally, when the transport layer cannot serve traffic demands, with the desired quality of service, the Xhaul controller asks the RL to negotiate with the radio layer a relaxation of the radio performances, e.g. coordination level. 


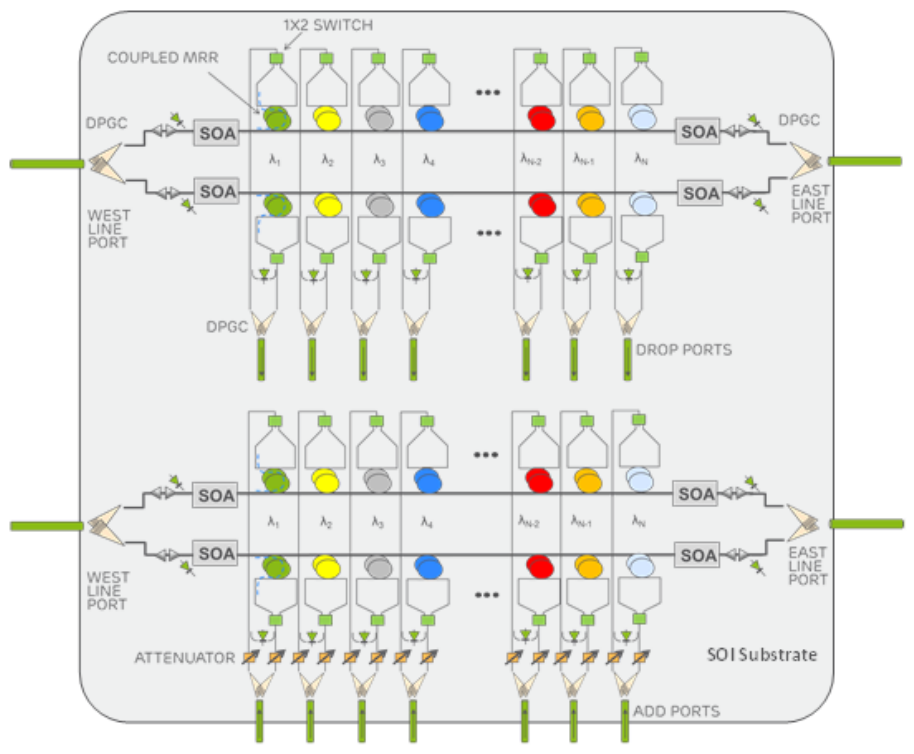

(a)

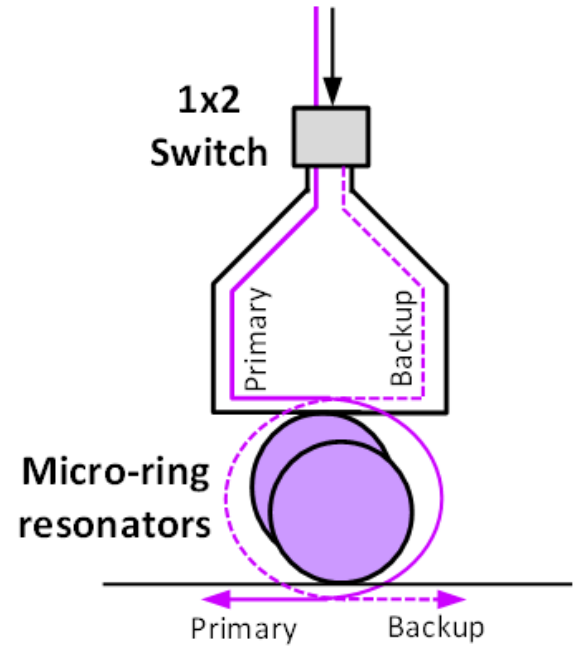

(b)

Fig. 9 - (a) Architecture of the Mini ROADM, (b) details of the switch elements

There are two main advantages with this approach: (i) it keeps separated radio and transport layers, while providing tight cooperation; (ii) operator-specific policies can be easily managed by introducing "administrative" parameters, enabling a multi-operator scenario.

The slicing scheme, implemented in the control architecture in Fig. 8, is based on Software Defined Networking (SDN) and Network Functions Virtualization (NFV) principles [9][10], but with specific peculiarities. For example, the south-bound interface is based on signaling protocols that are transmitted in band, exploiting available bits in the agnostic framing described in Section III.B and implemented in the demonstrator illustrated in Section V. This approach avoids a parallel and dedicated network for control

\section{EnABLING Photonic TeChNOLOGIES}

Xhaul calls for a new type of photonic devices with a much lower cost, a higher level of device miniaturization and a lower power consumption compared to current optical modules designed for metro or long haul.

Two types of devices are crucial for 5G optical transport: optical switches and multi-wavelength transceivers and photonic integration is definitely the enabling technology for both type of devices. Silicon photonics is most suitable for large scale integrated switching devices due to its characteristics of easy integration with control electronics, high miniaturization, mass-producibility, potential high yield and low cost due to use of well-established CMOS production infrastructure. As far as integrated multiwavelength transceivers are concerned relevant technological advances have been made with two different technological approaches: InP monolithic-integrated DWDM transceivers and silicon photonics based transceivers.

For optical switching a new type of system-on-chip device, referred in the following as Mini-ROADM, is under development in our labs and its architecture is depicted in Fig. 9 (a). A Dual Polarization Gain Control (DPGC) is followed by a Semiconductor Optical Amplifier (SOA) for the amplification of the DWDM comb. The DPGC adapt the random polarization of the input signal to the device main propagation mode. The polarization controller was experimentally characterized for 50 random polarization states. All the arbitrary polarization states were compensated within a $1 \mathrm{~dB}$ accuracy. Optical channels are added/dropped by a Silicon Micro Ring Resonators (MRR). The local channel ports are coupled to the ring by DPGCs and $1 \times 2$ switches that allows to revert the propagation direction and implement ring protection functionalities

In the prototype, the maximum drop loss difference from the first to the last channels is about $1.5 \mathrm{~dB}$. The measured channel isolation from adjacent channels is larger than 30 dB.

This device is especially designed for use in a double ring network with one ring transmitting downlink signals from the hub to the remote nodes and the other ring transmitting upstream signals from the remote nodes to the hub. This device is simpler than the conventional ROADMs used in metro networks. It has two line ports and 12 local ports to add and drop an equal number of $100 \mathrm{GHz}$ spaced optical channels. With the current Silicon Photonics technology, it is possible to expand the number of ports up to 24 while supporting a denser spacing (e.g. $50 \mathrm{GHz}$ ) would require technology advances to improve the wavelength stability of the micro-rings.

The fundamental functions performed by the MiniROADM are: DWDM channels multiplexing/de-multiplexing at DU site and reconfigurable add-drop of selected local channels at RRU site. Additional auxiliary networking functions have been added to the Mini-ROADM: channel 
direction switching and add-channel power regulation. The direction switching, that can be activate automatically, is implemented on each single wavelength for both add and drop functions and it is used to re-establish the connection in case of fiber break: when some of the nodes distributed along the ring become isolated, the wavelength signals used to communicate with those nodes change direction to overcome the fiber break. The fiber paths are then protected without the need to duplicate the optical interfaces at each add/drop port. Optical attenuators are implemented at the input of the add channel ports to regulate the optical power depending of the fiber span length: this can be beneficial to keep low the receiver sensitivity degradation due to crosstalk because it avoids that at the drop switch elements high power by-pass signals are transmitted together with the weak signal to be dropped.

For utilization of the Mini-ROADM in a real system it must be polarization insensitive and low loss, preferably lossless. Its design has been based on micro-ring resonators as wavelength selective switching elements[11][12][13], because of its small size (few $\mu \mathrm{m}$ ), low loss and low power consumption (few $\mathrm{mW}$ ).

The Mini-ROADM architecture presents two independent circuit structures one used for adding local channel to the network and one used for dropping channel from the network. Each circuit structure has a polarization diversity architecture in which the two TE and TM polarization components of the signals separated, at the device inputs, by a polarization splitter and rotator block (PSR) (implemented here by a double polarization grating coupler), are guided inside two separate optical buses to which a number of microring resonators are coupled. When the micro-ring resonator switches are activated the signals are dropped or added. At the output ports the two polarization components are recombined before coupling to the optical fibers.

The arrays of $1 \times 2$ optical switches at the input/output ports are used to provide the wanted bi-directionality to add and drop functions for each wavelength. Primary and backup paths are illustrated in Fig. 9 (b).

For supporting a data rate transmission up to 28 Gbaud an optical bandwidth of the micro-ring resonator switch element $>40 \mathrm{GHz}$ is specified and a two-coupled ring architecture has been implemented in order to provide the specified bandwidth together with the wanted adjacent channel isolation $(>20 \mathrm{~dB})$.

An array of optical attenuators is used to regulate the power of the added channels injected into the network and optical detectors are placed in strategic place inside the chip for monitoring.

Finally, III-V SOA dies are hybrid-integrated to the Silicon On Insulator (SOI) substrate in order to compensate for the device internal loss and fulfill as much as possible the lossless characteristics.

The Xhaul network also envisages compact, low power and low cost multi-wavelength transceivers.

InP monolithic integration technology [14] has made significant progresses and it is a possible candidate for the implementation of integrated multichannel DWDM TX and RX. Being based on the same material used today for producing discrete component it is mature for the realization of optical devices in the near terms while the monolithic integration of many channels in the same chip allows a cost reduction compared with the traditional discrete component approach.

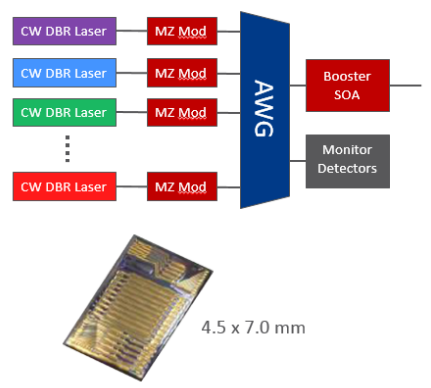

(a) (b)

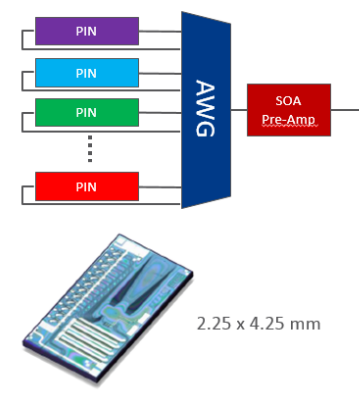

Fig. 10 - Realization of a InP-based monolithically integrated DWDM transceivers: (a) multi-channel transmitter, (b) multichannel receiver, Courtesy of Effect Photonics.

An example of implementation of a complete transceiver with $10 \mathrm{DWDM}$ channels, $100 \mathrm{GHz}$ spaced working at 10 Gbit/s in a CFP2 package, has been developed with the architecture shown in Fig. 10. The evolution toward $28 \mathrm{Gbit} / \mathrm{s}$ data rate is under development.

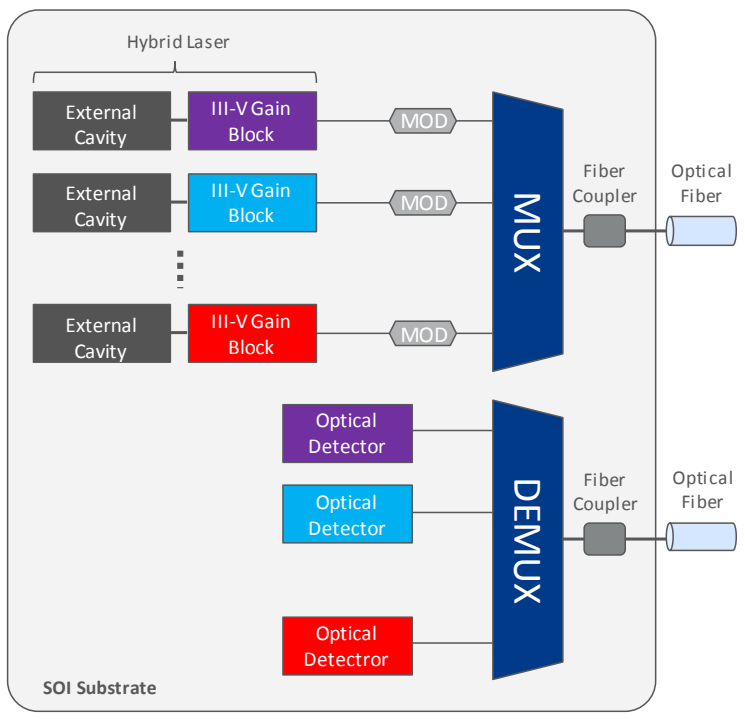

Fig. 11 - DWDM transceiver based on an array of hybrid lasers integrated with a silicon photonics chip.

Another technology which still needs some technological advances but promises, in a longer run, an even lower cost and higher level of miniaturization, is the hybrid laser technology based on the integration of III-V light generator dies with silicon photonic chips as shown in Fig. 11. With this approach all the optical circuits including laser external cavities, modulators, photodetectors are monolithically integrated in a SOI substrate, while the optical gain block is implemented in III-V material. The laser wavelength is set by controlling the wavelength selective elements inside the external cavity. Significant progresses toward the realization of low cost hybrid lasers in silicon photonics have been shown in [15][16] where the III-V integration is realized at wafer 
level without the need of an active alignment of each single light generator chip to the silicon substrate. These last developments indicate that a practical realization of low-cost, mass producible, high speed multi-channel DWDM transceivers for transmission up to $20 \mathrm{Km}$ in silicon photonic platform is realistic.

\section{PERFormance EVAluations}

Calculations to evaluate the performances have addressed the case of mixed BH and RPLI-based FH. A 5G Radio Access Technologies (RAT) has been considered with the following assumptions: $200 \mathrm{MHz}$ bandwidth in air, $125 \mu \mathrm{s}$ Time Transmission Interval (TTI), 256 QAM, beamforming (8x8 antennas). As a new functional split, it is assumed that the $5 \mathrm{G}$ radio unit is in charge of functionalities from physical layer to resource element mapping. With these hypothesis, the peak throughput for the RPLI interface is estimated in $34.1 \mathrm{Gbit} / \mathrm{s}$.

It is also assumed that the maximum tolerated end-to-end latency between radio units and baseband processing units is in the order of one TTI, that is $125 \mu \mathrm{s}$. In the Xhaul network, considering the uplink direction, this latency budget is spent for buffering/scheduling/framing in the remote node, for transmission in fiber, and for de-framing in the hub node. Similar considerations hold for downlink direction.

For illustrative purposes, it has been considered the case of a single $100 \mathrm{Gbit} / \mathrm{s}$ wavelength from the remote node to the hub node. The switch at the remote node, illustrated in Fig. 7, connects two 5G radio units, via RPLI, and Ethernet clients for RBS backhauling.

The buffer of the switch enables handling the unfortunate case of simultaneous arrivals of two radio traffic peaks from the two RPLI clients, delaying one of them of one TTI. Thanks to the buffer, the transport bandwidth for the transmission of the two RPLI clients is lower than the sum of the two traffic peaks, i.e. included between $34.1 \mathrm{Gbit} / \mathrm{s}$ and $68.2 \mathrm{Gbit} / \mathrm{s}$.

However, the use of a buffer is only possible if the fiber propagation delay doesn't absorb a significant portion of the end-to-end latency budget. If it is not possible to use the buffer, the transport bandwidth shall be dimensioned to the sum of the two peak, i.e. 68.2 Gbit/s.

In summary, the switch architecture in Fig. 7 allows to tradeoff between required transport bandwidth and latency.

In the $100 \mathrm{Gbit} / \mathrm{s}$ wavelength, the bandwidth which is not utilized for RPLI can be allocated for Ethernet BH traffic, which has not stringent latency constraints. Fig. 12 reports the bandwidth allocated for RPLI (blue line) and Ethernet (orange line), versus fiber link length.

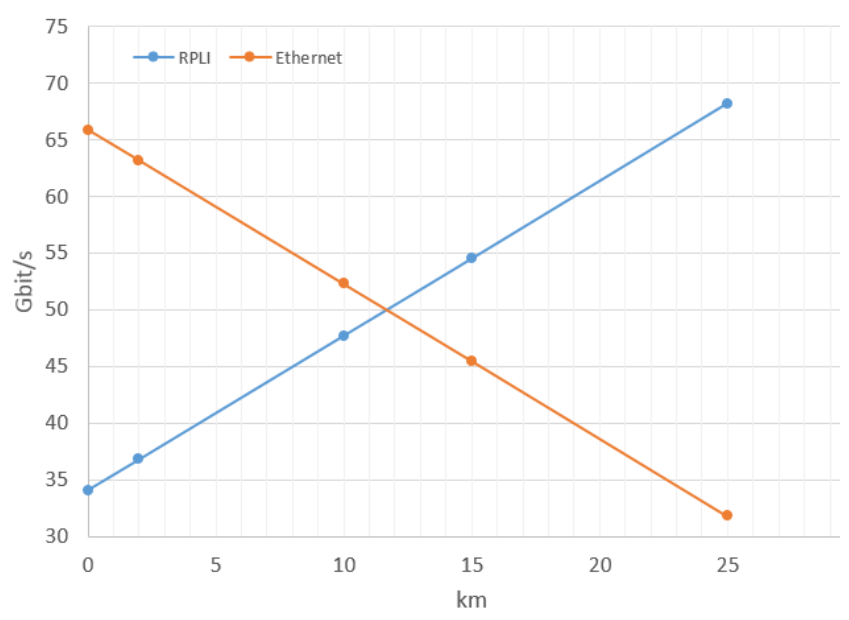

Fig. 12 - RPLI-based FH and BH transport bandwidth vs. fiber link length in a $100 \mathrm{Gbit} / \mathrm{s}$ wavelength

In the baseline case of $0 \mathrm{~km}$, RPLI can fully benefit from buffering so that a transport bandwidth of $34.1 \mathrm{Gbit} / \mathrm{s}$ is sufficient for the two clients. In the extreme case in which all the $125 \mu$ s end-to-end latency budget is absorbed by fiber propagation, $68.2 \mathrm{Gbit} / \mathrm{s}$ need to be allocated. This happens at $25 \mathrm{~km}$. In all the intermediate cases, the transport bandwidth required for the two RPLI clients linearly increase with distance.

As final remark, these results are an upper bound of the transport bandwidth for RPLI because it has been assumed that the radio traffic sources can peak simultaneously. A higher statistical multiplexing gain is expected in real scenarios.

\section{DEMONSTRATOR}

The Xhaul architecture, described in Section III, has found a first practical demonstration in an experimental test bed built at Ericsson Research labs. Purpose of the demonstrator is to test concurrent $\mathrm{BH}$ and $\mathrm{FH}$ transport and switching over dedicated or shared optical channels (Sections III.B and III.C, respectively) and the control architecture illustrated in Section III.D. Currently, the demonstrator uses state of the art optical technologies. The technologies described in sections III.A and IV will be included in the next release of the demonstrator.

The general topology illustrated in Fig. 2 is implemented in the test bed, schematically illustrated in Fig. 13. It is constituted by two remote nodes, located at the antenna side, and a hub node. Fig. 14 reports a picture of the demonstrator setup in lab. 


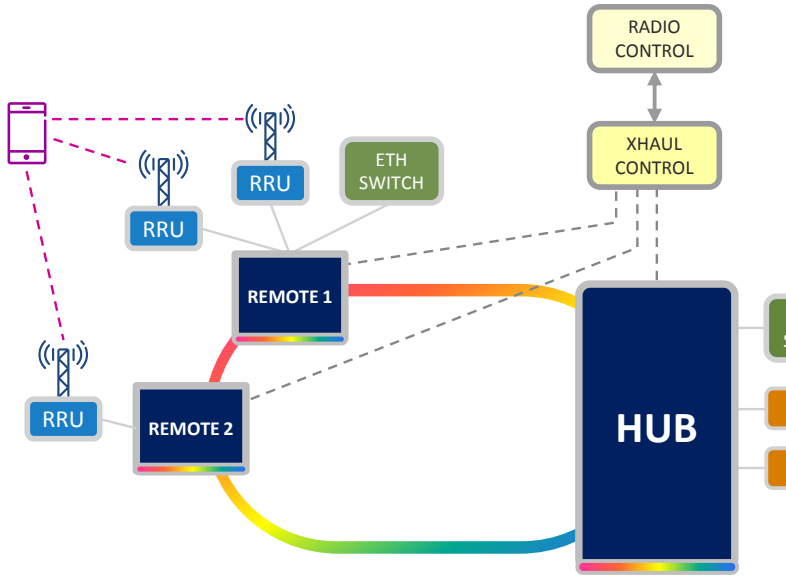

Fig. 13 - Sketch of the demonstrator setup

Two RRUs and an Ethernet Switch are connected to node Remote 1. One other RRU is connected to node Remote 2.

Each RRU generates a CPRI Option 3 (2.4576 Gbit/s) signal. The Ethernet switch generates two GbE signal to emulate BH signals from a RBS.

The signals, connected at each remote node are aggregated into a $10 \mathrm{Gbit} / \mathrm{s}$ wavelengths according to the framing protocol describes in section III-B and sent to the hub. The protocol includes Reed Solomon RS(255,239) FEC for Bit Error Monitoring, as well as a dedicated in-band OAM channel, Fig. 6. Limiting the number of interleaved codecs to 9 , the latency contribution, introduced by FEC, is $4 \mu$ s for each direction with no appreciable performance degradation, compared to standard OTN channels.

At each remote node, fixed OADMs are used for wavelength add/drop. The wavelengths are duplicated for protection. At the hub, Arrayed Waveguide Gratings (AWG) are used for wavelength multiplexing and de-multiplexing.

The ring is realized with double fiber, one for direction. The fiber span from Hub to Remote 1 is $14 \mathrm{~km}$; from Remote 1 to Remote 2 is $4 \mathrm{~km}$; and from Remote 2 to Hub is $6 \mathrm{~km}$.

The switch described in Section III.C is implemented by means of an Optical-Electrical-Optical structure. Referring to the upstream direction, for sake of simplicity, according to this structure, the $10 \mathrm{Gbit} / \mathrm{s}$ DWDM channels are: converted from optical to electrical; cross-connected by an agnostic crossbar matrix; and de-framed as described in section III-B. CPRI and Ethernet clients are sent to a CPRI mux/de-mux and to an Ethernet switch.

The DUs have their S1 interfaces connected to Core Network functions.

The Xhaul controller is realized with rack-mounted PC running Linux OS, connected to the hub via USB.

The demonstrator is able to showcase the following use cases:

1) Dynamic DU-RRU association

Following time-variant traffic loads, it is useful to consolidate baseband processing in a reduced number of DUs in low-load hours. Some DUs can be switched off with resulting power savings. To prove this use case, the Ethernet switch has been switched off and the CPRI traffic handled by Remote 1 has been

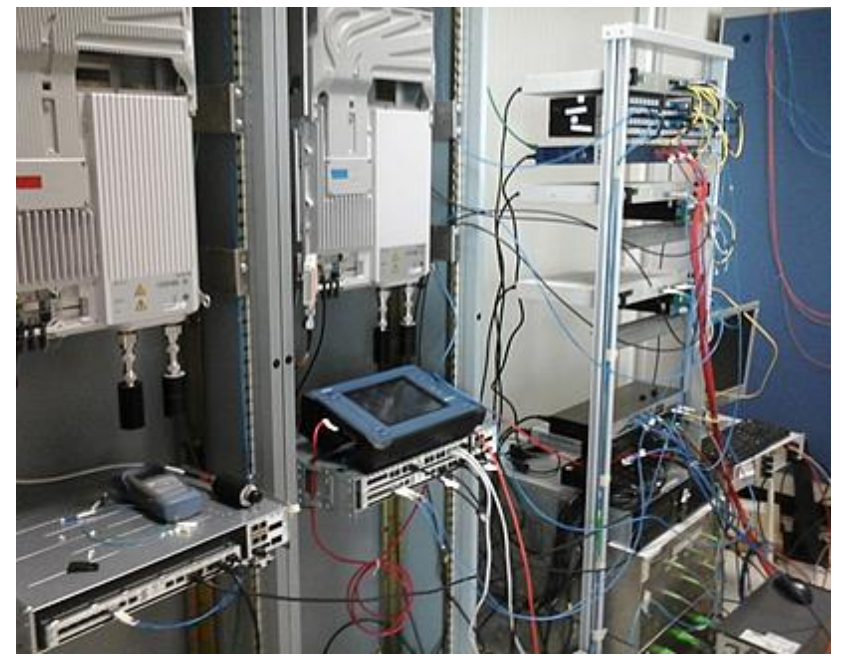

Fig. 14 - Picture of the demonstrator

moved to Remote 2 with no service interruption.

2) Transport links resiliency

Using FEC and pre-configured BER thresholds, a continuous monitoring of the transport link quality is performed and degradations are detected in a timely manner: before the number of errors become too high to be corrected by FEC, the protection path is activated. To emulate link degradation, a Variable Optical Attenuator (VOA) is placed in line.

3) $\mathrm{BH}$ and $\mathrm{FH}$ sharing the same optical channel

At Remote 1, Ethernet and CPRI are time-domain multiplexed in the same wavelength using the framing protocol described in III.B. No appreciable CPRI degradation, i.e. jitter, was detected due to the multiplexing with Ethernet.

4) Service slicing

The use case shows the capability to expose a sliced view of $5 \mathrm{G}$ services, hiding to users and to the service provider how said services are actually mapped on transport resources. This use case can be applied to share Xhaul infrastructure among different radio operators. As a practical proof, two video sources, generating GbE traffic, are connected to the Ethernet switch at Remote 1. As far as slicing of resources is concerned, these two clients are seen as two independent point to point Ethernet service by the service provider connected to the hub. The physical resource mapping of said slices is hidden to the upper layers. In the demonstrator, on Ethernet service is mapped on a wavelength shared with CPRI, the other one is transported by a dedicated wavelength on a different path.

5) Service-on-demand

The request of service can be done on demand and negotiated. In the demonstrator, the radio control asks to activate two CPRI Option 3 at Remote 1. The Xhaul controller, Fig. 8, checks the availability of bandwidth resources, with the required latency. If the check is passed, the video is activated. If not, e.g. the controller could not find a path with acceptable latency, less stringent requirements are proposed by the Xhaul controller. 


\section{CONCLUSIONS}

A Xhaul network architecture, based on optical technologies, agnostic time-deterministic switching and cooperative radio-transport control, has been discussed. The switch performance with new 5G functional split has been numerically assessed.

The Xhaul architecture has a logical hub and spoke topology which provides point-to-point logical connectivity, regardless of the physical network layout. It allows supporting significant 5G use case such as: dynamic association of DUs and RRUs; sharing of transport resources between $\mathrm{FH}$ and $\mathrm{BH}$; service slicing; and service provisioning on demand. A first practical experimental setup has been prepared in Ericsson Research labs, to demonstrate the use cases.

Xhaul, in the near future, can exploit novel photonic technologies which contribute to keep cost, power consumption and footprint low. Simulation and experiment have been presented.

\section{ACKNOWLEDGMENT}

The authors would like to thank Prof. Enrico Forestieri of Scuola Superiore Sant'Anna (Pisa) for the results in Fig. 3, Dr. Gianluca Meloni of Consorzio Nazionale Interuniversitario per le Telecomunicazioni (CNIT) and Dr. Francesco Fresi of Scuola Superiore Sant'Anna (Pisa) for the results in Fig. 4. 


\section{REFERENCES}

[1] NGMN "5G White Paper”, Feb. 2015 -

https://www.ngmn.org/uploads/media/NGMN_5G_White_Pape r_V1_0.pdf

[2] Ericsson, "Ericsson Mobility Report", Nov. 2015 http://www.ericsson.com/res/docs/2015/mobility-

report/ericsson-mobility-report-nov-2015.pdf

[3] Ericsson, "Cloud RAN", White Paper, Sep. 2015 https://www.ericsson.com/res/docs/whitepapers/wp-cloudran.pdf

[4] Common Public Radio Interface, "CPRI Specification version 7.0", Oct. 2015

[5] E. Forestieri and G. Prati, "Novel optical line codes tolerant to fiber chromatic dispersion," J. Lightwave Technol., vol. 19, pp. 1675-1684, Nov. 2001.

[6] ITU-T, "'Interfaces for the Optical Transport Network (OTN)", ITU-T Recommendation, Feb. 2012

[7] ITU-T, "G.Sup56 - OTN Transport of CPRI signals", Jul. 2015

[8] IEEE 802.1 Time Sensitive Networking (TSN), http://www.ieee802.org/1/pages/tsn.html

[9] D. McDysan, "Software defined networking opportunities for transport", IEEE Communications Magazine, vol. 51, issue 3, pp. 28-31, Mar.2013

[10] ETSI, "Network Functions Virtualisation (NFV); Architectural Framework", ETSI GS NFV 002, 01-2015.

[11] P.Contu, P Pintus, F. Testa, A. D'Errico and F. Di Pasquale, "Analysis and design of micro-ring based switch elements in silicon photonics for optical interconnection ", IEEE Optical Interconnect Conference 2013, Paper TuB3.

[12] P. Dong, W. Qian, H. Liang, R. Shafiiha, N.-N. Feng, D. Feng, X. Zheng, A. V. Krishnamoorthy, and M. Asghari, "Low power and compact reconfigurable multiplexing devices based on silicon microring resonators," Opt. Expr., vol. 18, no. 10, pp. 9852-9858, 2010.

[13] V. Sorianello, F. Testa, P. Velha, S. Doneda, M. Romagnoli, "Experimental evaluation of Residual Added Signal Crosstalk in a silicon photonics integrated ROADM", Proc. OFC 2014, Paper Th2A.30.

[14] M. Smit et al.: 'An introduction to InP-based generic integration technology', IOPscience, Semicond. Sci. Technol. 29 (2014) 083001 (41pp).

[15] G. Li, T. Creazzo, E. Marchena, P.K.L.Yu, and S. Krasulick,"A CMOS Wafer-Scale, Monolithically Integrated WDM Platform for TB/s Optical Interconnects", Proc. OFC 2014, Paper Th1C.2.

[16] Guang-Hua Duan et al.: "Hybrid III-V on Silicon Lasers for Photonic Integrated Circuits on Silicon", IEEE Journal of Selected Journal of Selected Topics in Quantum Electronics, , Vol. 20, No. 4, July/August 2014. 


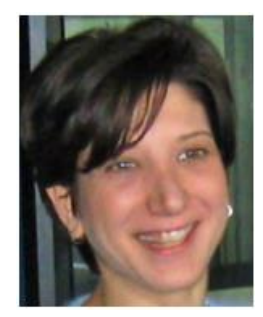

Paola Iovanna received her degree in electronics engineering from the University of Roma "Tor Vergata" in 1996. From 1995 to 1997 she collaborated with the FUB research center of Rome, working on fiber optic communications and optical networking. From 1997 to 2000 she worked at Telecom Italia, where she was involved in experimentation of new services based on different access technologies (e.g., XDSL, frame relay, optical). In 2000 she joined Ericsson in the Research Department where she dealt with networking and design solutions for packet and optical technology (GMPLS, MPLS, and Ethernet). From 2009 to 2012 she was responsible for carrying out research projects on packet and optical routing, control plane, and path computation solutions. In the framework of such activities she realized demonstrators and prototypes as well. In 2012 she was responsible for defining and prototyping SDN solutions for multi-domain transport in collaboration with customers. Since 2014 she has lead a research team to define transport networking and control solutions for 5G. She is actively involved in European projects and is on the Technical Program Committees of international conferences like ECOC. She holds more than 50 patents in routing, traffic engineering systems, and PCE solutions for packet-opto networks based on GMPLS, and multi-domain SDN transport, fronthaul, and backhaul solutions for $5 \mathrm{G}$, and is an author of several tens of publications in either international scientific journals or conferences.

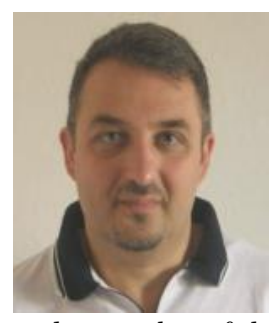

Fabio Cavaliere is with Ericsson, Italy, where he has been since 2006. As an expert in photonic systems and technologies, he contributes to developing strategy in optical transport and access networks. Before joining Ericsson, he was with Marconi, United Kingdom, from 1998 to 2006 in the Photonic Network and System Design Authority. He is coeditor of ITU-T Recommendation G.metro and a member of the Board of Stakeholders of Photonics 21. He has authored several publications and patents on optical communications.

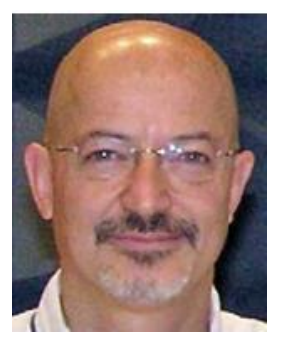

Francesco Testa received his degree in electronic Engineering, summa cum laude, from the University of Rome. In 1982 he was granted a scholarship from Fondazione Bordoni to work on integrated optics. In 1985 he worked at Alcatel-Face on coherent optical systems. He joined Ericsson in 1991 to work on the first demonstrations of WDM and later in research on architectures and technologies for optical networks. He is currently working as Principal Researcher at Ericsson Research focusing on photonics integrated technologies and applications.

Stefano Stracca received the MSc degree in Electronic Engineering from University of Rome in 1988. He joined Ericsson in 1990, assuming the role of $\mathrm{HW}$ and system designer for telecommunication products. Afterwards he held roles of technical coordinator, system manager, project manager and line manager in different product areas. Currently he is a Senior Researcher and deputy manager in Ericsson Research Optical Systems branch in Pisa, dealing mainly with optical networking solutions for 5G. He is also the Project Manager of European Commission FP7 STREP IRIS (Integrated Reconfigurable silicon photonic based optical Switch). He is inventor of several international patent filings.

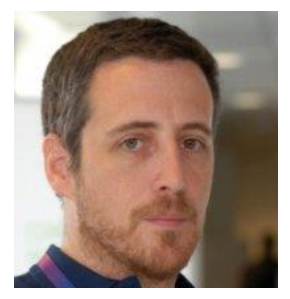

Giulio Bottari received telecommunication engineering degree from the University of Pisa in 1998. He then joined Marconi, Genoa, where he was a system designer of photonic communication equipment. Since 2006 he has been with Ericsson, Pisa. Currently he is a Senior Researcher and Technology Intelligence driver in Ericsson Research focusing on transport architectures for $5 \mathrm{G}$ radio network, IoT scenarios, and synchronization. He has filed 70 patents and is a co-author of 40 works in international referred journals and conferences.

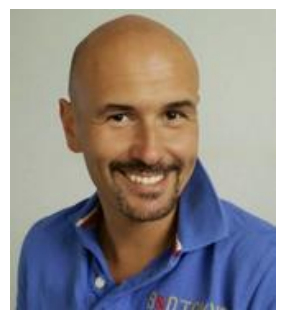

Filippo Ponzini was born in Piacenza, Italy, in 1973. He received a Master Degree in Telecommunications Engineering from University of Parma, Italy. He was a researcher in optical technologies with Scuola Superiore Sant'Anna, Pisa, Italy. Since 2007 he joined Ericsson Research. He is now mainly involved in optical networks and systems for radio access networks (RAN), in particular for radio heterogeneous networks and their evolution towards 5G. He is author of more than 30 publications and international patents.

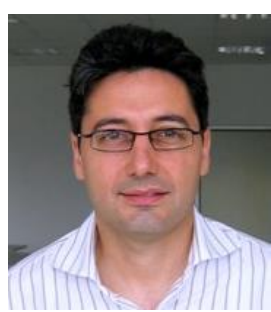

Alberto Bianchi received the Laurea degree in electronic engineering from the University of Pisa, Pisa, Italy. In 1998 he has been in electronic system department of University of Pisa. Since 1999, he has been with Ericsson Telecommunications, where he is now a Senior Engineer. From 1999 to 2006, he was in R\&D Laboratory, Rome, working on the HW design and the development of Ericsson Switch Systems. From 2006 to 2007, he was in R\&D Laboratory in Milan, working on radio MINI-LINK products. In 2007 he joined Ericsson Research in Pisa. His interests are in the design of optical systems and networks, photonics switching based on integrated photonics, mobile backhaul/fronthaul networks and Silicon Photonics applications. He has authored several publications and patents.

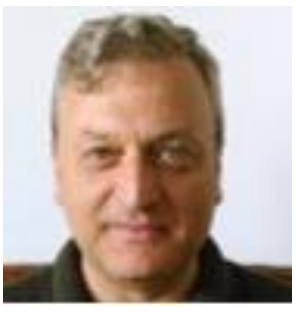

Roberto Sabella is manager of the Italian branch of Ericsson Research. He got his D.Eng. Degree in electronic engineering in 1987, then joined Ericsson where he gained experience in packet-optical networks and technologies, traffic engineering and routing, and telecommunications networks. He has authored more than 100 papers for international journals, magazines, and conferences, as well as two books on optical communications. He holds more than 20 patents and has been an adjunct professor at the Sapienza University of Rome. He has guest edited many special issues in several journals and magazines. 